\title{
TASA DE INFECCIÓN DE LA PUDRICIÓN DEL TALLO EN MAÍZ CAUSADA POR F usarium moniliforme ${ }^{1}$
}

\author{
Mariano Mendoza Elos ${ }^{2}$, Enrique Andrio Enríquez ${ }^{2}$, Alfonso López Benítez ${ }^{3}$, Raúl Rodríguez Guerra ${ }^{4}$, \\ Luis Latournerie Moreno ${ }^{5}$, Sergio A. Rodríguez Herrera ${ }^{3}$
}

\section{RESUMEN}

Tasa de infección de la pudrición del tallo en maíz causada por Fusarium moniliforme. El objetivo de éste trabajo fue determinar la resistencia horizontal de 134 líneas $\mathrm{S}_{1}$ de maíz al hongo Fusarium moniliforme. La enfermedad fue inducida mediante inoculación en la etapa de floración masculina bajo condiciones de campo utilizando la técnica del palillo. Las líneas se evaluaron en México en 2002. La estimación de la enfermedad se hizo en dos ocasiones con una separación de 20 días. Las evaluaciones mostraron que las líneas Roque-801, 804, 1103 y 1405 fueron resistentes en la primera evaluación. Las tasas de incremento de la enfermedad variaron de 0,01 a 0,229 por unidad por día. Las líneas mostraron bajos niveles de enfermedad de 0 a $1 \%$ en la primera evaluación. En la segunda evaluación, los niveles de enfermedad variaron de 49 a $100 \%$. En un programa de mejoramiento genético estas líneas pueden ser utilizadas para mejorar la resistencia horizontal del maíz a la pudrición del tallo causada por Fusarium moniliforme.

Palabras clave: Nivel de enfermedad, línea, resistencia, Fusarium moniliforme, Zea mays L.

\begin{abstract}
Infection rate of the stalk rot in maize to Fusarium moniliforme. The objective of this work was to determine the horizontal resistance of $134 \mathrm{~S}_{1}$ lines of maize to Fusarium moniliforme. The lines were evaluated in Mexico during 2002. The disease was induced by inoculation at male flowering time under field conditions using the technique of the thud pick. Assessment of disease data was done twice with a time interval of 20 days. Field evaluations showed that lines 302 and 802 were resistant in the first and second evaluations. Estimated rates of disease increase varied form 0.018 to 0.299 per unit per day. Lines $110,302,706,801$, 1104 showed low levels of disease from 19 a $52 \%$ in the first evaluation. In the second evaluation, disease levels ranged from 39 a $79 \%$. Rates of infection increase for these lines were $0.074,0.051,0.043,0.69$, and 0.088 , respectively. In a maize breeding program these lines could be utilized to improve F. moniliforme and stalk rot horizontal resistance.
\end{abstract}

Key words: Rate of disease, line, resistance, Fusarium moniliforme, Zea mays $\mathrm{L}$.

\section{INTRODUCCIÓN}

Las pudriciones de tallo en el maíz son causados por dos géneros de hongos: Gibberella y Fusarium
(Jugenheimer 1988), y dentro de este último género se tienen como especies a moniliforme y graminearum (De León 1984), éstas se caracterizan considerando la fase del cultivo en la que se desarrollan. Fusarium spp.

Recibido: 8 de abril, 2005. Aceptado: 2 de febrero, 2006.

2 Instituto Tecnológico de Roque. Apdo. Postal 508, Km. 8 Carr. Celaya-Juventino Rosas, Roque, Celaya, Guanajuato, México CP 38110. Correspondencia: mmendoza66@hotmail.com

3 Departamento de Fitomejoramiento, Universidad Autónoma Agraria Antonio Narro, Buenavista, Saltillo, Coahuila, México CP25315.

4 Profesor investigador del INIFAP, Km 6.5 Carretera Celaya-San Miguel, Campo Celaya, Gto.

5 Instituto Tecnológico de Konkal. Apdo. Postal 53 “D” Col. Itzimma. C.P. 97100. Conkal, Yucatán. 
es una enfermedad fúngica de cereales, que infecta frecuentemente: trigo (Triticum vulgare), cebada (Hordeum vulgare), avena (Avena sativa), centeno (Secale cereale), maíz (Zea mays) y pastos forrajeros. F. moniliforme, es capaz de colonizar maíz durante todo el ciclo vegetativo de la planta incluyendo las siguentes partes de la planta: raíz, tallo, mazorca y semillas. En muchos casos, la presencia del hongo, no es perceptible, pues no causa daño visible en la semilla o en la plántula (Singh et al. 1977; Thomas y Buddenhagen 1980). Esta infección asintomática puede presentarse en cualquier parte de la planta y ser transmitida por la semilla a las plántulas, pudiéndose o no desarrollar una infección sistémica (Munkvold y Desjardins 1997). Los granos de maíz son altamente vulnerables a la degradación por micotoxinas; se presentan principalmente en condiciones climáticas secas y calurosas, por daño de los insectos a la mazorca y debido a los bajos niveles de resistencia (Agrios 2001).

La infección de mazorcas por este hongo, es importante debido al potencial tóxico de sus metabolitos secundarios, las fumonisinas; substancias carcinogénicas secretadas por el hongo en los granos de las mazorcas afectadas. Las cepas de F. moniliforme producen varias toxinas y compuestos con acción fitohormonal, entre las cuales se encuentran el ácido fusárico, fusarinas (fusarina $\mathrm{C}$ ), giberelinas, moniliformina, zearalenona, tricotecenos y fumonisinas (Nelson 1992; Bacon y Williamson 1992; Sweeney y Dobson 1998). Estas últimas, fueron reportadas y caracterizadas por investigadores en Sudáfrica como un agente promotor de cáncer hepático en ratas (Gelderblom 1988). Las fumonisinas fueron inicialmente asociadas a leucoencefalomalacia y duodenitis en equinos y después en micotoxicosis en cerdos, pollos y en cáncer esofágico en humanos (Miller et al. 1995; Nelson et al. 1993; D'Mello et al. 1999).

En México se presentan pérdidas por la pudrición de tallo y grano causado por Fusarium, este hongo aparece primero como una coloración salmón pálido en el pedicelo o casquete de la punta de los granos. Eventualmente, los granos infectados muestran un crecimiento de moho polvoso de color rosáceo, compuesto por grandes números de esporas o conidias. Las líneas puras difieren en su resistencia a esta enfermedad. Las líneas puras susceptibles tienen "estigmas cortados" o granos reventados. Tales líneas transmiten tegumentos débiles de la semilla a sus híbridos y tienden a ser susceptibles a la pudrición del grano por Fusarium. El hongo penetra cerca de la corona de la planta o a través de los nudos. Las plantas afectadas se vuelven cafés y los tejidos se reblandecen en la parte inferior del tallo. Conforme avanza la enfermedad, el patógeno se distribuye en el tejido medular y sólo quedan en el tallo las fibras de los vasos que conducen el agua (Jungenheimer 1988).

La resistencia es una característica heredable y controlada principalmente por el sistema genético nuclear y en algunos casos por el citoplasma. La teoría de la resistencia vertical y horizontal se basa en estimar la tasa de infección del patógeno o la tasa de incremento de la enfermedad. La tasa de incremento afecta la expresión de la resistencia vertical y determina el beneficio de este tipo, retrasando el inicio de la enfermedad, en el caso de la horizontal afecta la tasa de incremento de la enfermedad determinándose así el efecto sobre una epifitia (Van der Plank 1984). Se han identificado fuentes de resistencia para muchas de las enfermedades de importancia económica cuyos genes se encuentran presentes dentro del genoma del maíz. Algunos autores definen la resistencia como la capacidad de la planta para reducir el crecimiento y desarrollo del patógeno o parásito después que ha habido contacto entre el hospedante y el patógeno o después que éste ha iniciado su desarrollo o se ha establecido (Niks et al. 1993).

Las líneas puras difieren considerablemente en su resistencia a Fusarium, por lo que el mejor método de control es el uso de híbridos. En este sentido, Mendoza et al. (2003) determinaron para esta enfermedad el componente genético que indica la presencia de efectos maternos no significativos, presencia de genes aditivos y dominantes, observándose una heredabilidad de 0,11 , la cual es considerada como baja, por lo anterior, se propone como objetivo la estimación de la tasa de infección en la pudrición del tallo de maíz por Fusarium moniliforme.

\section{MATERIALES Y MÉTODOS}

El presente trabajo se realizó en el campo experimental del Instituto Tecnológico de Roque. Ubicado en el kilómetro 8 de la Carretera Celaya-Juventino Rosas en el año 2002; cuyas coordenadas son $20^{\circ} 32^{\prime}$ de latitud norte y $100^{\circ} 45^{\prime}$ de latitud oeste, a una altitud de 1762 msnm (Erazo 2000). Para la región Celaya, los principales climas son semi-cálido, sub-húmedo $\mathrm{BS}$, y $\mathrm{C}$ (Wo) con temperatura media que oscila de los $14^{\circ}$ a $22^{\circ} \mathrm{C}$ y precipitación que varían de 600 hasta $1.000 \mathrm{~mm}$ anuales. Los suelos prominentes en la región son Vertisol Pélico y Vertisol Crómico, con predominancia de los primeros. Estos son de textura arcillosa, planos de reacción ligeramente alcalina, sumamente fértiles y aptos para una gran variedad de cultivos. El material genético utilizado fueron 134 líneas S1 provenientes de una población mejorada de polinización libre. 
El trazo y siembra del experimento se realizó el 29 de junio del 2002, y se sembró de forma manual depositando 21 semillas en parcelas de surcos de $5 \mathrm{~m}$ de longitud, a una distancia entre surcos de $0,75 \mathrm{~m}$ y entre plantas $0,20 \mathrm{~m}$. La fórmula de fertilización que se utilizó fue $200-80-00$, que equivale a $367 \mathrm{~kg} / \mathrm{ha}$ de urea (46-0000) y $174 \mathrm{~kg} / \mathrm{ha}$ de fosfato monoamónico. Se aplicó el 50 $\%$ de nitrógeno y el $100 \%$ de fósforo al momento de la siembra y el otro $50 \%$ restante en la segunda escarda (18-46-00). La obtención del inóculo se realizó en el laboratorio del INIFAP, éste se obtuvo a partir de granos de maíz infestados por Fusarium provenientes de una colecta de maíz que se hizo en el estado de Guanajuato. En este trabajo fueron observados $F$. moniliforme y $F$. solani de acuerdo a la clave de identificación de especies a $F u$ sarium (Samuels et al. 2001).

Para el aislamiento del hongo se utilizó la metodología descrita por Agrios (2001). Los granos de maíz se colocaron en cámaras húmedas, utilizando para ello, charolas de plástico estériles, papel sanita y agua destilada. Los granos fueron sometidos a tratamiento en una solución de hipoclorito de sodio al 5\% por un minuto, se lavaron con agua destilada para eliminar el exceso de la solución. Luego, se colocaron las sanitas en la parte inferior de las charolas, se pusieron 100 granos y se roció con agua destilada, a los ocho días se observó el crecimiento del hongo. Una vez desarrollado el hongo, se realizó un cintazo para identificarlos en el microscopio, y posteriormente transferirlos de manera aséptica a cajas petri con medio de cultivo PDA (papa, dextrosa, agar) acidulado con el fin de evitar contaminación por el desarrollo de bacterias. El incremento del hongo se realizó en palillos con PDA. En cajas petri esterilizadas se colocaron aproximadamente 100 palillos (lavados y esterilizados) en capas de 25 , colocadas en forma perpendicular, se envolvieron en papel y se esterilizaron nuevamente. Previamente se preparó medio de cultivo PDA, y al enfriarse, se vació en las cajas de petri con los palillos esterilizados sin llenar. Posteriormente, con una jeringa se rociaron los palillos con inóculo. A las seis semanas se observó abundante desarrollo de micelio y esporulación.

La técnica de inoculación utilizada fue la del taladro y palillo recomendada por Coutiño (1973) como la más apropiada para trabajos de pruebas de resistencia a pudriciones de tallo. Se utilizó un taladro de pilas recargable, con brocas de 3/32”. La metodología consistió en taladrar el primer entrenudo con el taladro y broca mencionada, enseguida se introdujo un palillo portador del inóculo y se identificó la planta con pintura en aerosol. Las inoculaciones se realizaron en floración el día 7 y 24 de septiembre de 2002, la cual ocurrió entre los 69-82 días después de la siembra; las inoculaciones realizadas se efectuaron en un máximo de ocho plantas.
Para identificar estas inoculaciones, a las plantas de la primera inoculación se les marco con pintura en aerosol roja y a las segundas con azul.

Se realizaron dos evaluaciones por inoculación, estas se hicieron a los 20 días después de depositado el hongo en la planta, para ello la evaluación fue destructiva, ya que se cortaron los tallos longitudinalmente para observar el avance del daño y darle la calificación correspondiente. La escala utilizada fue la descrita por Coutiño (1973):

\section{Escala para daños}

Grado 1, no existe desarrollo alrededor del punto de inoculación $(0 \%)$.

Grado 2, existe poca invasión de tejido (25\% de entrenudo dañado).

Grado 3, cuando el daño ha cubierto el 50\% del entrenudo.

Grado 4, cuando hay el $75 \%$ invasión del entrenudo inoculado.

Grado 5, cuando el hongo invade los entrenudos próximos al inoculado (100\%).

Las plantas con la escala de 1, se consideraron como resistentes, aquellas que obtuvieron grado dos se tomaron como tolerantes, las plantas con grado tres moderadamente tolerante, el grado cuatro moderadamente susceptible y el grado cinco como susceptibles. La tasa de incremento de la enfermedad se estimó con la siguiente fórmula (Van der Plank 1984):

$$
r=\frac{2.3}{t_{1}-t_{2}} \log _{10} \frac{x_{2}\left(1-x_{1}\right)}{x_{1}\left(1-x_{2}\right)}
$$

Donde:

$r$, es la tasa de incremento de la enfermedad.

$x_{1}$, es la cantidad de enfermedad al momento de la primera evaluación.

$x_{2}$, es la cantidad de enfermedad al momento de la segunda evaluación.

$t_{1}$, fecha de la primera evaluación.

$t_{2}$, fecha de la segunda evaluación.

2.3 , valor constante.

$t_{1}-t_{2}=20$

\section{RESULTADOS Y DISCUSIÓN}

Las tasas de infección proporcionan en una sola cifra, información con respecto al hospedante, al patógeno 
y las condiciones ambientales en las que progresa la enfermedad. En la primera lectura (20 días después de la inoculación), se encontraron valores de daño a la enfermedad que oscila desde 0 hasta 100 , indicaron niveles de resistencia, tolerancia y moderada tolerancia a completa susceptibilidad. Sólo se encontraron cuatro líneas resistentes, la Roque-801, 804, 1103 y 1405 . Con valor de dos y que se refiere a las líneas tolerantes fueron 10. El resto (120 tratamientos) resultaron con grados de tres, cuatro y cinco que fueron calificadas de moderadamente tolerantes hasta susceptibles. Este último germoplasma queda descartado a futuro para integrarse al programa de mejoramiento de maíz. En la segunda evaluación, algunas de estas líneas (804, 808, 902, 1.103, 1.108, $1.405,1.710,1.801)$ mostraron una completa susceptibilidad con niveles de enfermedad de $100 \%$, mostrando las más altas tasas de infección (de 0,261 a 0,299). Las tasas de infección variaron de 0,018 a 0,299, con una media de 0,145 , una desviación estándar de 0,131 y un intervalo de confianza de 0,020 (Cuadro 1). El rompimiento del control de las epifitas mediante variedades con resistencia de genes mayores, ha conducido a los fitomejoradores a reevaluar la resistencia horizontal, utilizando métodos de evaluación, como la estimación de las tasas de infección de las enfermedades en el campo. La selección de materiales con resistencia horizontal midiendo la tasa aparente de infección (r) ha sido utilizada en varias especies. En el Instituto de Investigaciones Agronómicas de cultivos tropicales se estimó la tasa de infección de Pyricularia oryzae en 30 variedades de arroz, y ésta varió de 0,115 a 0,692 , siendo el promedio de $0,258 \pm 0,142$ y los menores valores de $\mathrm{r}(0,60,0,65$, 0,73 ) fueron para las variedades resistentes (Dhan 1986).

En otro grupo de líneas, (101, 205, 305, 502, 1.004, $1.204,1.209,1.308,1.310,1.410$ y 1.703$)$, el porcentaje de daño de la enfermedad en la primera evaluación fue relativamente alto, variando de 72 a $90 \%$ y, en la segunda evaluación el porcentaje de daño de la enfermedad para estas mismas líneas varió de 79 a $100 \%$. Sin embargo, las tasas de infección fueron bajas. La explicación para esto fue que las condiciones del hospedante y de crecimiento del patógeno cambiaron, para el momento en que se realizó la primera evaluación ya

Cuadro 1. Promedio de daño causado por Fusarium moniliforme en un grupo de líneas de maíz evaluadas en la localidad de Celaya, Guanajuato, México. 2002.

\begin{tabular}{|c|c|c|c|c|c|c|}
\hline $\begin{array}{c}\text { Número } \\
\text { de línea } \\
\text { (Progenitor) }\end{array}$ & Origen & $\begin{array}{c}1 \text { era } \\
\text { Evaluación } \\
\text { X1 }\end{array}$ & $\begin{array}{c}\text { Porcentaje } \\
\text { De daño } \\
\text { X1 }\end{array}$ & $\begin{array}{c}2 \text { da } \\
\text { Evaluación } \\
\quad \text { X2 }\end{array}$ & $\begin{array}{c}\text { Porcentaje } \\
\text { De daño } \\
\text { X2 }\end{array}$ & $\begin{array}{c}\text { Tasa } \\
\text { Crecimiento } \\
\mathbf{r}\end{array}$ \\
\hline 8 & Roque-110 & $2,0^{2}$ & 25 & $4,0^{4}$ & 80 & 0,074 \\
\hline 16 & Roque-302 & 2,0 & 25 & 3,0 & 75 & 0,051 \\
\hline 23 & Roque-406 & 2,0 & 25 & 4,5 & 90 & 0,116 \\
\hline 34 & Roque-601 & 1,5 & 12 & 4,5 & 90 & 0,140 \\
\hline 36 & Roque-603 & 2,0 & 25 & 4,5 & 90 & 0,113 \\
\hline 45 & Roque-705 & 1,5 & 12 & $5,0^{5}$ & 100 & 0,273 \\
\hline 49 & Roque- 801 & $1,0^{1}$ & 0 & 2,5 & 33 & 0,069 \\
\hline 52 & Roque-804 & 1,0 & 0 & 5,0 & 100 & 0,299 \\
\hline 55 & Roque- 808 & 2,0 & 25 & 4,5 & 90 & 0,126 \\
\hline 57 & Roque-902 & 2,0 & 25 & 5,0 & 100 & 0,251 \\
\hline 59 & Roque-904 & 4,5 & 90 & 5,0 & 100 & 0,105 \\
\hline 66 & Roque-1004 & $5,0^{3}$ & 100 & 5,0 & 100 & 0,092 \\
\hline 70 & Roque-1010 & 4,5 & 90 & 5,0 & 100 & 0,125 \\
\hline 72 & Roque-1102 & 4,5 & 90 & 5,0 & 100 & 0,105 \\
\hline 73 & Roque-1103 & 1,0 & 0 & 5,0 & 100 & 0,299 \\
\hline 74 & Roque-1104 & 2,0 & 25 & 4,0 & 75 & 0,088 \\
\hline 80 & Roque-1202 & 4,5 & 90 & 5,0 & 100 & 0,105 \\
\hline 90 & Roque-1310 & 5,0 & 100 & 5,0 & 100 & 0,092 \\
\hline 94 & Roque-1405 & 1,0 & 0 & 5,0 & 100 & 0,299 \\
\hline 99 & Roque-1502 & 5,0 & 100 & 5,0 & 100 & 0,092 \\
\hline 115 & Roque-1710 & 1,5 & 12 & 5,0 & 100 & 0,261 \\
\hline 116 & Roque-1801 & 1,5 & 12 & 5,0 & 100 & 0,273 \\
\hline \multirow[t]{2}{*}{126} & Roque-1909 & 2,0 & 25 & 5,0 & 100 & 0,237 \\
\hline & & & & & Media & $0,145^{*}$ \\
\hline
\end{tabular}

*, promedio de las 134 líneas evaluadas.

${ }^{1}$ Los tratamientos con calificación de uno, se consideraron como resistentes, 2 aquellas que obtuvieron grado dos se tomaron como tolerantes, ${ }^{3}$ las plantas con grado tres moderadamente tolerante, ${ }^{4} \mathrm{el}$ grado cuatro moderadamente susceptible y $5 \mathrm{el}$ grado cinco fueron tomadas, como susceptibles. 
existía un alto nivel de enfermedad, quedando poco tejido susceptible por infectar. Cuando se realizó la segunda lectura, la enfermedad solo avanzó sobre ese limitado tejido susceptible. En términos epidemiológicos, cuando la resistencia de una variedad a una enfermedad se expresa uniformemente contra todas las razas del patógeno, se dice que es resistencia "horizontal" y, no implica interacción diferencial entre patógeno y hospedante. Su estabilidad es atribuible a su naturaleza poligénica, actúa reduciendo la efectividad de uno o mas de los componentes del parasitismo del patógeno a través de la acumulación de genes menores que actúan cuantitativamente en el hospedante (Van der Plank 1984).

Un tercer grupo de líneas (110, 302, 706, 801, 1.104) mostró en la primera evaluación relativamente bajos niveles de enfermedad que variaron de 19 a $52 \%$, indicando una respuesta de resistencia a moderada tolerancia, y en la segunda evaluación, los niveles de enfermedad variaron de 39 a $79 \%$, indicando la misma respuesta de resistencia a moderada tolerancia, las tasas de infección fueron $0,074,0,051,0,043,0,69,0,088$, respectivamente. Nelson (1978) expresa que, la tasa de incremento de una enfermedad o simplemente la tasa de infección, es la tasa con la cual incrementa la población de un patógeno en un cultivo. La resistencia horizontal reduce la tasa aparente de infección. Van der Plank (1984) describe que la resistencia horizontal poligénica disminuye el avance de las epifitas después de que se han iniciado, por lo que uno puede determinar el efecto de la resistencia horizontal en el desarrollo de una enfermedad por su efecto en la tasa de infección. Parlevliet (1983) observó que cuando las variedades de trigo con resistencia horizontal y las variedades sin ella, crecen unas al lado de las otras en el campo, y son uniformemente inoculadas con razas a las que todas las variedades son susceptibles, la cantidad de infección difiere.

\section{CONCLUSIONES}

Las tasas de incremento de la enfermedad variaron de 0,01 a 0,229 por unidad por día. La tasa promedio de avance de la enfermedad fue 0,145 por día. Del grupo de líneas sometido a evaluación sólo se encontraron cuatro líneas resistentes en la primer evaluación. En la segunda evaluación, los niveles de enfermedad variaron de 49 a $100 \%$. En un programa de mejoramiento genético estas líneas pueden ser utilizadas para mejorar la resistencia horizontal del maíz a la pudrición del tallo causada por Fusarium moniliforme.

Cabe señalar, que también es posible obtener tasas de infección bajas en materiales susceptibles cuando las evaluaciones de la enfermedad se hacen en la parte superior de la curva de desarrollo de la enfermedad, porque la cantidad de tejido susceptible disponible para la infección ya es limitado. Este no es el caso para estas últimas líneas.

\section{LITERATURA CITADA}

AGRIOS, G.N. 2001. Fitopatología. 2 ed. Limusa. México. $838 \mathrm{p}$.

BACON, C.W.; WILLIAMSON, J.W. 1992. Interactions of Fusarium moniliforme, its metabolites and bacteria with corn. Mycopathology 117:65-71.

COUTIÑO, A. A. 1973. Evaluación de siete técnicas de Inoculación de hongos causantes de pudriciones en tallos y mazorcas de maíz. Tesis profesional. Escuela Superior de Agricultura "Hermanos Escobar". Cd. Juárez, Chihuahua. $71 \mathrm{p}$.

DHAN P. S. 1986. Breeding for resistance to diseases and insect pest . Spring- Verlag, Berlin Heidelber New York London Tokio. p. 222.

DE LEÓN, C. 1984. Enfermedades del maíz, guía para su identificación en campo. 3 ed. CIMMYT. México. 114 p.

D’MELLO, J.P.; PLACINTA, C.M.; MACDONALD, A.M. 1999. Fusarium mycotoxins: A review of global implications for animal health, welfare and productivity. Animal Feed Science and Technology 80:183-205.

ERAZO, B. H. 2000. Resistencia y daño de pulgones en el rendimiento y calidad de la semilla de trigo (Triticum aestivum L.). Tesis de Maestría, Instituto Tecnológico Agropecuario No. 33. Celaya, México. 100 p.

GELDERBLOM, C.A.; JASKIEWICZ, K.; MARASAS, F.O.; THIEL, P.G.; HORAK, R.M.; VLEGGAAR, R.; KRIEK, P.J. 1988. Fumonisins - novel mycotoxins with cancer-promoting activity produced by Fusarium moniliforme. Applied and Environmental Microbiology 54:1806-1811.

JUGENHEIMER, R. W. 1988. Maíz, variedades mejoradas, métodos de cultivo y producción de semillas. Limusa, México. p. 841.

MENDOZA, E.M.; LÓPEZ, B.A.; MORENO, M.E.; DE LEÓN, C. 2003. Herencia genética y citoplasmática de la mazorca del maíz (Zea mays L.) causada por Fusarium moniliforme Sheld. Revista Mexicana de Fitopatología 21 (3): 267-271. 
MILLER, J.D.; SAVARD, M.E.; SCHAAFSMA, A.W.; SEIFERT, K.A.; REID, L.M. 1995. Mycotoxin production by Fusarium moniliforme and Fusarium prolife from Ontario and ocurrence of fumonisin in the 1993 corn crop. Canadian Journal of Plant Pathology 17:233-239.

MUNKVOLD, G.P.; DESJARDINS, A.E. 1997. Fumonisins in maize. Can we reduce their occurrence? Plant Disease 81:556-563.

NELSON, R.R. 1978. Genetics of horizontal resistance to plant diseases. Annu. Rev. Phytopathol. 16:359-379.

NELSON, P.E. 1992. Taxonomy and biology of Fusarium moniliforme. Mycopathology 117:29-36.

NELSON, P.E.; DESJARDINS, A.E.; PLATTNER, R.D. 1993. Fumonisins, mycotoxins produced by Fusarium species: Biology, chemistry and significance. Annu. Rev. Phytopathol. 31:233-252.

NIKS, R.E.; ELLIS, P.R.; PARLEVLIET, J.E. 1993. Resistance to parasites. In M.D. Hayward, N.O. Bosemark, I. Romagosa eds. Plant breeding: principles and prospects. Chapman \& Hall, London, England. p. 422-447.

PARLEVLIET, J. E. 1983. Can horizontal resistance be recognized in the presence of vertical resistance in plants exposed to a mixture of pathogen races? Phytopathology 73:379.

SAMUELS, G..J.; NIRENBERG, H.I.; SEIFERT, K.A. 2001. Perithecial species of Fusarium. pp.1-14. In: B.A. Summerell; J.F. Leslie; D. Backhouse; W.L. Bryden; L.W. Burgess eds. Fusarium. Paul E. Nelson Memorial Symposium. The American Phytopathological Society. St. Paul, Minnesota, USA. 392 p.

SINGH, D.; SINGH, T. 1977. Location of Fusarium moniliforme in kernels of maize and disease transmission. Indian Journal of Mycology and Plant Pathology 7:32-38.

SWEENEY, M.J.; DOBSON, D.W. 1998. Mycotoxin production by Aspergillus, Fusarium and Penicillium species. International Journal of Food Microbiology 43: 141-158.

THOMAS, M.D.; BUDDENHAGEN, I.W. 1980. Incidence and persistance of Fusarium moniliforme in symptomless maize kernels and seedlings in Nigeria. Mycologia 72:882-887.

VAN DER PLANK, J. E. 1984. Disease resistance in plants. $2^{\mathrm{a}}$ ed. Academic Press. N. Y., London, San Francisco. 194 p. 